\title{
ON THE JACOBIAN CRITERION OF FORMAL SMOOTHNESS
}

\author{
ANTONIO G. RODICIO
}

\begin{abstract}
We give a short proof of the jacobian criterion of formal smoothness using the Lichtenbaum-Schlessinger cotangent complex.
\end{abstract}

The aim of this note is to get a proof of the following jacobian criterion of formal smoothness:

Theorem 1. Let $A$ be a ring, $B$ a noetherian A-algebra, $J$ an ideal of $B$, and $C=B / J$. Let us consider over $A$ and $B$ the discret and $J$-adic topology, respectively. Then, the following statements are equivalent

1) The A-algebra $B$ is formally smooth

2) For every representation $B \simeq R / I$, where $R$ is a smooth $A$-algebra, the canonical homomorphism

$$
I / I^{2} \otimes_{B} C \longrightarrow \Omega_{R \mid A} \otimes_{R} C
$$

is left invertible.

This theorem has been obtained by $M$. André [1, prop. 16.17] using simplicial methods. We shall get a more elementary proof, based on the LichtenbaumSchlessinger (co-)homology theory [3], and a counter-example showing that this result is not true for arbitrary $B$.

First we recall the definition of the Lichtenbaum-Schlessinger (co-)homology functors. Let $A$ be a ring, $B$ an $A$-algebra and $M$ a $B$-module. Choose a polynomial algebra $R$ over $A$ such that $B \simeq R / I$, and a free $R$-module $F$ such that there exists an exact sequence of $R$-modules

$$
0 \longrightarrow U \longrightarrow F \stackrel{j}{\longrightarrow} I \longrightarrow 0 .
$$

Let $U_{0}$ be the image of the homomorphism $\phi: F \otimes_{R} F \rightarrow F, \phi(x \otimes y)=$ $j(x) y-j(y) x$ and consider the complex of $B$-modules

$$
L_{B \mid A}: 0 \longrightarrow U / U_{0} \longrightarrow F / I F \longrightarrow \Omega_{R \mid A} \otimes_{R} B \longrightarrow 0 .
$$


Then, $T_{i}(B \mid A, M)=H_{i}\left(L_{B \mid A} \otimes_{B} M\right), T^{i}(B \mid A, M)=H^{i}\left(\operatorname{Hom}_{B}\left(L_{B \mid A}, M\right)\right)$, $i=0,1,2$.

There exist isomorphisms: $T_{0}(B \mid A, M) \simeq \Omega_{B \mid A} \otimes_{B} M, T^{0}(B \mid A, M) \simeq$ $\operatorname{Der}_{A}(B, M) \simeq \operatorname{Hom}_{B}\left(\Omega_{B \mid A}, M\right), T^{1}(B \mid A, M) \simeq \operatorname{Exalcom} A(B, M)$ the set of equivalence classes of infinitesimal extensions of $B$ over $A$ by $M$, and $T^{ \pm}(B \mid A, M)$ $\simeq \operatorname{Hom}_{B}\left(I / I^{2}, M\right)$ if $B \simeq A / I$.

Proposition 1. Let $A$ be a ring, $B$ an $A$-algebra, $J$ an ideal of $B$ and $C=B / J$. Assume that $B \simeq R / I$, where $R$ is a smooth $A$-algebra. Then, the following conditions are equivalent

1) The canonical homomorphism $I / I^{2} \otimes_{B} C \rightarrow \Omega_{R \mid A} \otimes_{R} C$ is left invertible

2) $T_{1}(B \mid A, C)=0$ and $\Omega_{B \mid A} \otimes_{B} C$ is a projective $C$-module

3) $T_{1}(B \mid A, M)=0$ for all $C$-module $M$.

Proof: Since $R$ is a smooth $A$-algebra, we have $T_{1}(R \mid A,-)=0=T^{1}(R \mid A,-)$ [3, prop. 3.1.3]. Hence there exist exact sequences [3, 2.3.5]

$$
\begin{array}{r}
0 \longrightarrow T_{\mathrm{I}}(B \mid A, C) \longrightarrow I / I^{2} \otimes_{B} C \longrightarrow \Omega_{R \mid A} \otimes_{R} C \longrightarrow \Omega_{B \mid A} \otimes_{B} C \longrightarrow 0 \\
0 \longrightarrow \operatorname{Hom}_{C}\left(\Omega_{B \mid A} \otimes_{B} C, M\right) \longrightarrow \operatorname{Hom}_{C}\left(\Omega_{R \mid A} \otimes_{R} C, M\right) \longrightarrow \\
\operatorname{Hom}_{C}\left(I / I^{2} \otimes_{B} C, M\right) \longrightarrow T^{1}(B \mid A, M) \longrightarrow 0,
\end{array}
$$

where $M$ is a $C$-module.

The result follows from this sequences having into account that $\Omega_{R \mid A} \otimes_{R} C$ is a projective $C$-module [3, prop. 3.1.3].

For every $C$-module $M$, the homomorphisms $A \rightarrow B \rightarrow B_{n}=B / J^{n}$ induce an exact sequence

$$
T^{1}\left(B_{n} \mid B, M\right) \longrightarrow T^{1}\left(B_{n} \mid A, M\right) \longrightarrow T^{1}(B \mid A, M) \longrightarrow T^{2}\left(B_{n} \mid B, M\right)
$$

and, therefore, an exact sequence

$$
\begin{aligned}
& \stackrel{\lim }{\longrightarrow} T^{1}\left(B_{n} \mid B, M\right) \longrightarrow \underline{\lim } T^{1}\left(B_{n} \mid A, M\right) \longrightarrow T^{1}(B \mid A, M) \\
& \longrightarrow \stackrel{\lim }{\longrightarrow} T^{2}\left(B_{n} \mid B, M\right)
\end{aligned}
$$

The formal smoothness of $B$ over $A$ is equivalent; by [2, prop. 19.4.4], to the vanishing of $\lim T^{1}\left(B_{n} \mid A, M\right)$ for all $C$-module $M$. Then, Theorem 1 is a consequence of Proposition 1 and

Proposition 2. Let $A$ be a noetherian ring, $I$ an ideal of $A, A_{n}=A / I^{n}$, and $M$ an $A / I-$ module. Then

i) $\underset{\lim }{\longrightarrow} T^{1}\left(A_{n} \mid A, M\right)=0$.

ii) $\underset{\lim }{\longrightarrow} T^{2}\left(A_{n} \mid A, M\right)=0$. 
Part i) is easy: $\quad \lim _{\longrightarrow} T^{1}\left(A_{n} \mid A, M\right) \simeq \stackrel{\lim }{\longrightarrow} \operatorname{Hom}_{A / I^{n}}\left(I^{n} / I^{2 n}, M\right) \simeq$ $\lim _{\longrightarrow} \operatorname{Hom}_{A / I}\left(I^{n} / I^{n+1}, M\right)=0$.

To prove part ii) we need the following lemmas.

Lemma 1. Let $A$ be a ring, $I$ an ideal of $A, B=A / I$, and $M$ a $B$-module. Then, there exists a natural monomorphism of $B$-modules $T^{2}(B \mid A, M) \rightarrow$ $\operatorname{Ext}_{A}^{1}(I, M)$.

Proof: Let $F$ be a free $A$-module such that there exists an exact sequence of $A$-modules $0 \rightarrow U \rightarrow F \stackrel{j}{\rightarrow} I \rightarrow 0$. Let $U_{0}$ be the image of the homomorphism $\phi: F \otimes_{A} F \rightarrow F, \phi(x \otimes y)=j(x) y-j(y) x$. Then, $T^{2}(B \mid A, M)=$ $\operatorname{Coker}\left(\operatorname{Hom}_{A}(F, M) \rightarrow \operatorname{Hom}_{A}\left(U / U_{0}, M\right)\right)$. The result follows from the diagram of exact sequences

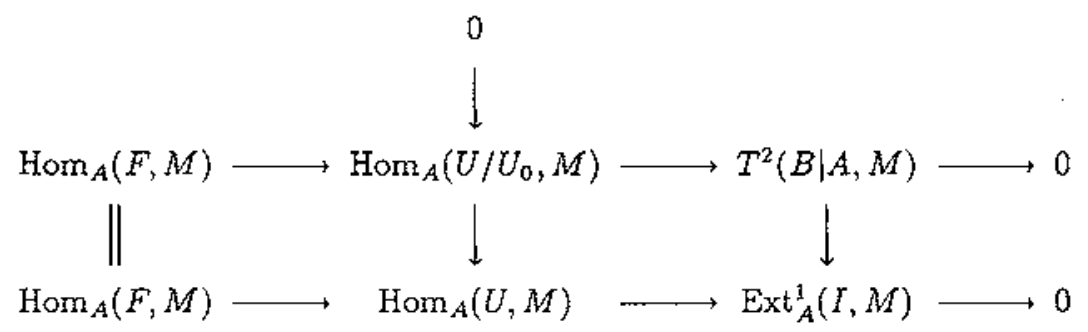

Lemma 2. Let $A$ be a noetherian ring, $I$ an ideal of $A$ and $n>0$ an integer number. Then, there exists $s \geq n$ such that the canonical homomorphism

$$
\operatorname{Tor}_{1}^{A}\left(I^{s}, A / I\right) \rightarrow \operatorname{Tor}_{1}^{A}\left(I^{n}, A / I\right)
$$

is trivial.

Proof: We have $\operatorname{Tor}_{1}^{A}\left(I^{n}, A / I\right) \simeq \operatorname{Tor}_{2}^{A}\left(A / I^{n}, A / I\right) \simeq \operatorname{Tor}_{1}^{A}\left(A / I^{n}, I\right)$. Let $0 \rightarrow U \rightarrow F \rightarrow I \rightarrow 0$ be an exact sequence of $A$-modules where $F$ is free and of finite type. Then

$$
\operatorname{Tor}_{1}^{A}\left(A / I^{n}, I\right) \simeq\left(U \cap I^{n} F\right) / I^{n} U .
$$

By Artin-Rees lemma $\left[4\right.$, th. 15] there exists $r>0$ such that $I^{t} F \cap U=$ $I^{t-r}\left(I^{r} F \cap U\right)$ for $t>r$. Taking $s=n+r$ we obtain $I^{s} F \cap U \subseteq I^{n} U$ and therefore

$$
\operatorname{Tor}_{1}^{A}\left(I^{s}, A / I\right) \longrightarrow \operatorname{Tor}_{1}^{A}\left(I^{n}, A / I\right)
$$

is trivial.

We now prove part ii) of Proposition 2. By lemma 1 there exists a monomorhism

$$
\stackrel{\lim _{\longrightarrow}}{\longrightarrow} T^{2}\left(A_{n} \mid A, M\right) \longrightarrow \lim _{\longrightarrow} \operatorname{Ext}_{A}^{1}\left(I^{n}, M\right) .
$$


On the other hand, for each $n$ we have an exact sequence

$$
0 \rightarrow \operatorname{Ext}_{A / I}^{1}\left(I^{n} / I^{n+1}, M\right) \rightarrow \operatorname{Ext}_{A}^{1}\left(I^{n}, M\right) \rightarrow \operatorname{Hom}_{A / I}\left(\operatorname{Tor}_{1}^{A}\left(I^{n}, A / I\right), M\right)
$$

which is deduced, for instance, from the change-rings spectral sequence

$$
E_{2}^{p, q}=\operatorname{Ext}_{A / I}^{p}\left(\operatorname{Tor}_{q}^{A}\left(I^{n}, A / I\right), M\right) \Rightarrow \operatorname{Ext}_{A}^{p+q}\left(I^{n}, M\right)
$$

Since $\lim _{\longrightarrow} \operatorname{Ext}_{A / I}^{1}\left(I^{n} / I^{n+1}, M\right)=0$ and $\lim _{\longrightarrow} \operatorname{Hom}_{A / I}\left(\operatorname{Tor}_{1}^{A}\left(I^{n}, A / I\right), M\right)=0$, by lemma 2, we obtain $\stackrel{\lim }{\longrightarrow} \operatorname{Ext}_{A}^{1}\left(I^{n}, M\right)=\overrightarrow{0}$. Therefore, $\underset{\longrightarrow}{\lim } T^{2}\left(A_{n} \mid A, M\right)=0$.

Theorem 1 is not true for arbitrary $B$. To exhibit a counter-example, we need the following result.

Lemma 3. Let $A$ be a ring and $I \subseteq T$ two ideals of $A$ such that $T^{2}=T$ and $I T \neq I$. Let $B=A / I, J=T / I$ and $C=B / J$. Then, the A-algebra $B$ is formally smooth for the $J$-adic topology, but there exists a $C$-module $M$ such that $T^{1}(B \mid A, M) \neq 0$.

Proof: We have $J^{2}=\left(T^{2}+I\right) / I=(T+I) / I=T / I=J$. Hence, for each $C=A / T$-module $M$

$$
\varliminf_{\longrightarrow} T^{1}\left(B_{n} \mid A, M\right) \simeq T^{1}(C \mid A, M) \simeq \operatorname{Hom}_{A / T}\left(T / T^{2}, M\right)=0
$$

where $B_{n}=B / J^{n}$. Therefore, $B$ is formally smooth.

On the other hand $T^{1}(B \mid A, M) \simeq \operatorname{Hom}_{B}\left(I / I^{2}, M\right) \simeq \operatorname{Hom}_{A / T}(I / I T, M)$. Since $I / I T \neq 0$, we obtain $T^{3}(B \mid A, I / I T) \neq 0$.

Counter-example. $A=C(R, R)$ the ring of all real-valued continous functions on $R, I=$ principal ideal of $A$ generated by the identity function, and $T=$ maximal ideal of $A$ containing $I$ (see [5], Ch. $2, \S 2$, Ex. 15).

This counter-example solves negatively a question of A. Brezuleanu [6, Remark $1.3(\mathrm{i})]$.

\section{References}

1. M. ANDRE, "Homologie des Algebres Commutatives," Springer, 1974.

2. A. Grothendieck, EGA $0_{I V}$, Publ. Math. IHES 20, Paris, 1964.

3. S. LICHTENBAUM, M. SCHLESSINGER, The cotangent complex of a morphism, Trans AMS 128 (1967), 41-70.

4. H. Matsumura, "Commutative Algebra," Benjamin/Cummings, 1980. 
5. A. BREzuleanu, Smoothness and regularity, Compositio Math. 24(1972), $1-10$.

Departamento de Algebra

Facultad de Matemáticas

15771 Santiago de Compostela

SPAIN

Rebut el 12 de Setembre de 1988 